\title{
REPRESENTATION OF SYNTHETIC SHADOWS ON 3D DIGITAL MODELS FOR THE VOLUMETRIC INTERPRETATION ON CONCAVE-CONVEX ARCHAEOLOGICAL ARTIFACTS
}

\author{
J. Garcia-Fernandez ${ }^{1 *}$, L. Medeiros² ${ }^{2}$ J. Pernão ${ }^{1}$ \\ ${ }^{1}$ Faculdade de Arquitetura, Universidade de Lisboa, Portugal - (jorgefernandez, jnpernao)@fa.ulisboa.pt \\ ${ }^{2}$ Faculdade de Ciências Sociais e Humanas, Universidade Nova de Lisboa, Portugal - leonormedeiros@fcsh.unl.pt
}

Commission II

KEY WORDS: Representation, Interpretation, Archaeology, Shadows, 3D modelling

\begin{abstract}
:
The representation of archaeological artefacts aims for the graphic description of relevant information from the object, to allow for the proper interpretation of evidences from the past. Concavities and convexities are elements often difficult to represent through classical (analogical) representation techniques, especially when these geometrical characteristics are neither continue nor parameterizable. Digital techniques have advanced on the accurate reconstruction of 3D shapes, while attaching real colour to the geometry. However, the perception of concave/convex shapes from photorealistic true-orthoimages continues to be limited, especially when rich and homogeneous textures camouflage slight slope changes or volumetric deformations. In this paper we first critically review the current practice on the representation of solid-of-revolution artefacts with concave-convex predominance, and alternative photorealistic representations aiming at a better understanding of volume and colour. Given the limitation targeted, we then propose a workflow for the creation of true-orthometric maps enhanced by customized shadows. The work-flow integrates considerations on: (i) The orientation of archaeological artefact; (ii) the creation of accurate orthometric images based on Digital photogrammetry techniques; and (iii) the application of synthetic attached and cast shadows according the shape (information) to be represented. The workflow is demonstrated with a sample of plates retrieved from the Rua-das-Madres archaeological site, in Portugal.
\end{abstract}

\section{INTRODUCTION}

The drawing of archaeological finds has been, since the beginning of archeological practice, a fundamental method for the understanding of the material culture associated with past societies. The 18 th and early 19 th century illustrations, heirs of the rigueur developed in the Renaissance, were crucial for the communication of archaeological work and showed a growing tendency for the scientific-eye and the creation of methodologies. But it will be the work promoted by processual archeologists in the 1950s, with their focus on methodology, that will focus on transforming the representation into a normalized action. Today, as digital technologies have entered the day-to-day life, and as hand-drawing of archaeological artifacts is becoming a lost practice, new methods can bring renewed opportunities in the process of representation of these materials towards increasing comprehension of material culture.

Technical drawing in archaeology, as applied to artefacts, today follows a set of rules of representation (although with variations according to the school or author), that are focused on the codes that should be applied to the representation of different materials and to different types of objects; see, (Adkins, Adkins 1989), (Griffiths, Jenner, Wilson, 1990), (Lima, 2007), and (Madeira, 2013), for an overview. The drawing of ceramic artifacts has been a key element of focus for this discussion (see Castro, Sebastian, 2003 for an example) because they are the most common typology found in

\footnotetext{
* Corresponding author
}

archaeological digs, usually in vast numbers and providing good indication for chronology and livelihood.

Several codes are applied to the representation of ceramics, as are certain assumptions about the object. When we handle containers (from cups to plates) we assume its symmetry by imagining a vertical axis on the center of the object; the rotation of the fragment on that axis allows us to define the profile of the artefact (as a surface of revolution). With the shape identified, the final drawing will represent the object (or its reconstruction if using a fragment), oriented in its position of use, as if set on a horizontal plane, at the eye-level of the observer. This allows for the easy identification of the object, complemented by the representation of its profile and its thickness. The drawing has two main areas: on the let of the axis we see the section and the inside of the object, and on the right, we see the profile and exterior view of the object. If any of these areas has some type of decoration or relevant evidence, it is represented in the according space. Often, especially in the case of plates with decorations, a top perspective (and sometimes also a bottom one) must be represented. So, although we can infer the shape from the first type of representation, in the presence of decorations it also requires the inclusion of another perspective of the object, placed above, as in the folding of the top plane in the American system of representation of projections.

This means that a decision is done in every drawing, by the archaeologist, of what to represent and what to omit, which is 
then reflected in the amount of information offered. For example, while a decoration will be represented, fractures or evidences of the potter's wheel, or marks of the support of the plate when it is glazed, may be left out if it is felt that their inclusion will cloud the representation.

This set of codes, briefly presented here, when used, contains all the necessary information for the archaeologist to understand the piece depicted: the dimensions, the shape (typology), the decorations and/or details of construction and use, and the amount of piece that has survived (fragment vs complete piece). This understanding derives from the training and experience the archaeologist possesses on this interpretation, but the question remains on whether this representation is equally clear to the less trained eye, for example in a museum exhibit or nonacademic publication. In these platforms we see increasingly favored the use of replicas, digital models, photographs, or artistic reproductions, distant from the archaeological scientific representation that is often in their base.

In drawing, the use of shadows is a common way to aid in the interpretation of the volume of the artefact, a crucial information in the understanding of both open shapes (like plates) and closed shapes (like vases). Even if its depiction is often dispensable - when the main goal of the drawing is to clarify general typologies - if used, then light is defined as being on the left, in a $45^{\circ}$ angle in relation to the horizontal work plane. As a result, the object will have areas of shadow, represented through dotting, with its intensity varying accordingly to the amount of light that would reach certain areas of the object.

One of the reasons why shadows are usually omitted from the drawing of an archaeological artefact is that, given the limited amount of symbols that can be used, reduced to the use of the line and the dot, adding shadows can conflict with the representation of decorations and of the expression of the type of material (for example, dotting is common in the representation of hand-made ceramics of pre and proto historic periods, to show the lack of homogeneity in the ceramic paste, so the use of more dots to represent the shadow that the object casts on itself would add complexity to the understanding surface).

The collection of objects used for this study originated from an archaeological intervention at Rua das Madres, in Lisbon (Fig. 1), which identified an area of discard of pieces from a ceramic oven that was active in the 17 th and 18 th centuries (Manso, Medeiros, Kruz, 2011). The artefacts collected were mostly representative of both finished (glazed) and semifinished (unglazed) products, namely plates. Of these, the ones without glaze (the blue over white decorations nationally known as faiança) still contain a rich expression of elements and provide indications for the way they were made, namely differentiating the use of potter's wheel, used here, from the use of molds, which leaves other traces in the paste. In typical representations these plates would be used to assess typologies, not considered for detailed drawing in favor of the glazed ones. As such, their details (the marks of the turning of the potters' wheel, the fingerprints of the potter and the pressure areas that slightly distort the perfect form of the piece), on this site and on the many that are constantly being identified in the city, are largely left un-explored and unrepresented. They present although an opportunity to look at these pieces and provide analytical perspectives that aren't overshadowed by the drawing of decorations.

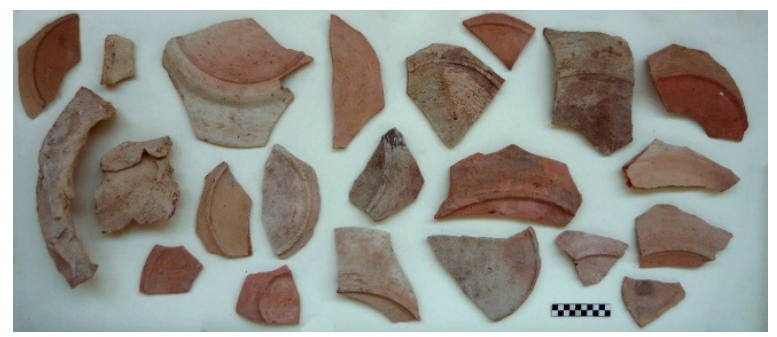

Figure 1: Sample of collected artifacts in the archaeologist site Rua das Madres, Portugal

If we eliminate the variable of having the representation of shadows hinder the reading of painted decorations or relevant features, we can take advantage of the potential of $t$ new digital methodologies in 'reading' the object in itself. The new level of magnification and detail that digital tools bring, and which extend the capacities of the human hand and eye, offer new possibilities for the identification and understanding of the details mentioned above. While the trained eye can look for clues that provide information on the fabrication, usage or accidents that the material may have gone through, digital representations make them clearer and even bring up details impossible to be identified by the naked eye. Since these details mainly imply an abrupt change in the continuity of the surface, the digital reading of concave-convex variations in archaeological material provides not only new information but an alternative way of representing it.

\section{DIGITAL PROCESS OF 3D RECONSTRUCTION}

Our strategy to enhance the lecture/interpretation of concaveconvex artifacts, first require an accurate and dense digital 3D information of the piece of study.

Unlike the $3 \mathrm{D}$ reconstruction based on active sensors (Laser Scanning, SAR, Radar), photogrammetric procedures don't depend on a unique equipment but in a combination of the camera configuration and the reconstruction algorithms. This implies a greater capacity in the variation of the working scales (far and close ranges). Moreover, since the input data for photogrammetry-supported reconstruction (digital Photos) contains a reliable information of the scene's colors, the output information display an accurate representation of the real texture (in term of color). Considering the relevance of both geometrical and radiometrical information to understand the evidence of the past in archeological artifacts, we decided to conduct the digital reconstruction using Photogrammetric methods.

The 3D reconstruction based on images, (image-based information) has been already proved as one a main tool within the documentation of Cultural Heritage (García-Fernández, 2014). During the last few years, supported by the improvement of both devices and software, digital photogrammetry has experienced a vertical evolution. While digital cameras and lenses have increased the performance whilst decreasing their prices, it has been the digital evolution of software and formats what has solidly democratized its use.

New capacities on massive pixel correspondences, thought the implementation of Dense Stereo Matching algorithms (DSM), have aimed to find global and local correspondence (between pixels and disparities in the vicinity of the pixel), and to densify the sparse results to a dense correspondence-point cloud. A 
variety of approximation have been implemented based on DSM, from solutions based on areas, to solutions based on characteristics (assigning attributes of position, shape, lengths, and stablishing relations of proximity, intersection and adjacency between similar characteristics). More recently, with the addressed Structure from Motion on a reverse engineering strategy (Martorelli, Pensa, Speranza, 2014), no prior information of the internal information is needed to solve the relative orientation of the camera. Both internal and relative parameters are solved at the same time, reducing or eliminating the need of on-site calibration.

\subsection{Spatial Resolution}

In photogrammetry, the spatial resolution is giving by the size of the footprint of the minimum unit (pixel) of the sensor on the object, also known as ground sample distance (GSD). Since the photographic camera (and its optic system) is the main tool to capture the input for 3D reconstruction of the physical object, its configuration determines the spatial resolution. This depends on the distance to the object; the combination of size of the sensor and number of pixels; and the focal length (distance between the lens and the sensor).

For the demonstration of the here-presented flow, it was used a NIKON 7200 camera, sensor APS-C, and a fixed lens Nikon AF Micro Nikkor $60 \mathrm{~mm} \mathrm{f} / 2.8 \mathrm{D}$, allowing us to focus to lifesize. In our case the working distance ranged from $90-100 \mathrm{~mm}$, having an average DSD of $0.06 \mathrm{~mm}$. The estimated GSD corresponds to a single measurement on the object, measured at the most favorable point. This leads to "optimistic" values of the GSD for the rest the object.

To undertake the small Depth-of Field obtained on the aforementioned configuration, we carried the creation of a single image through a relational calculus of the periodic and continues variation of the focus (Niederoest, Niederoest, Scucka, 2004). Using a shape-from focus method, this process was able not only to create a fully focused image (Fig. 2, right), but a depth map (Fig. 2 left), useful for background masking and assessment of the focusing on the scene. The data was processed using the software Agisoft Photoscan Pro. The references for the external orientation where extracted from the piece using a digital caliper (accuracy in measures of $0.02 \mathrm{~mm}$ up to $150 \mathrm{~mm}$ )

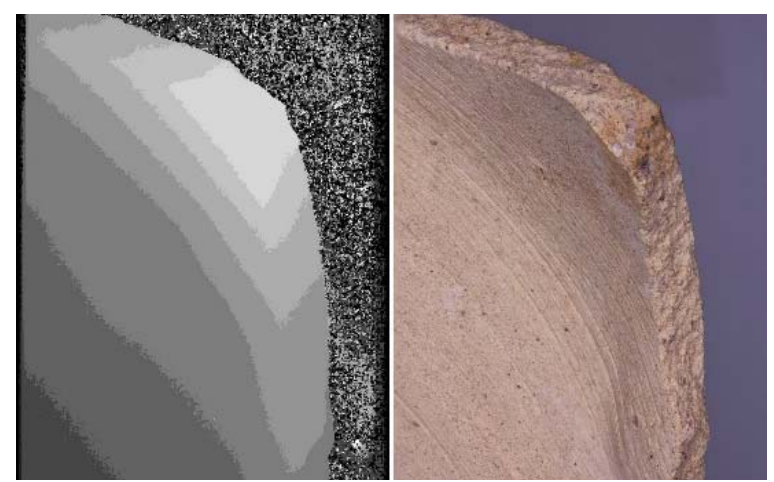

Figure 2: Compiling photos based on stacked focus create a depth map (left); and a final fully-focused image (right)

\subsection{Extraction of measures for the orientation of the piece}

Once the piece is $3 \mathrm{D}$ reconstructed, it becomes necessary to obtain basic geometric information to orient the artifact, meeting the requirements defined in the previous section.

For the specific case of solid of revolution such as the case of study, it was measured: the axis of revolution, 2D Shape of the principal section, and radius of the main concentric rings. Fig. 4 represents the use of these values for the representation of the cross section at the eye-level, and both top \& bottom orthometric true-color representation.

\section{LIGHT, SHADOW AND VOLUME}

Light is the genesis of our visual perception. We infer shapes and volumes from the colours we receive through our sight sense and organized in the perception/cognition processes of our brain. Light and shadow are the two correlated elements that inform our perception of the tridimensional characteristics of objects (Swirnoff, 1986). There is much scientific research on the shape from shadow concept (Ullman, S., 1996).

In volume recognition, intrinsic (attached) and extrinsic (cast) shadows are the most important elements in the process. Intrinsic shadows are responsible for directly infer illuminant tilt, qualitative global surface structure, and, at intersections with surface creases, the concavity/convexity of a surface. Cast shadows on the background, or on other objects, inform us on the form of the object that blocks the light, the shape of the background or of the other object where the shadow is cast on and the position of the object in space (if the object is in the air the cast shadow will not contact with the object).

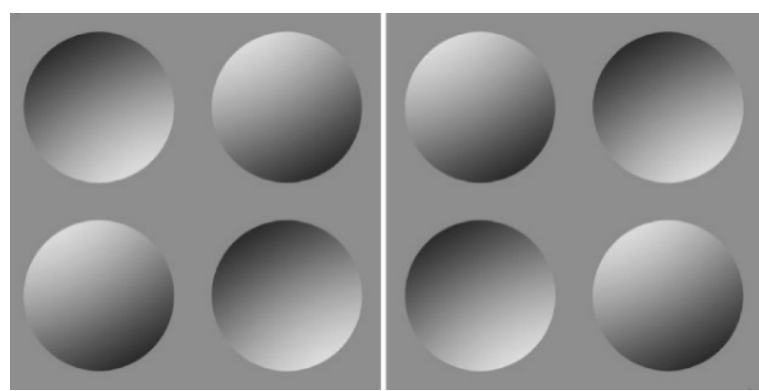

Figure 3: Convex and concave perception based on light direction, from (Stone et al., 2009)

From our life's perception experience, we infer some properties out of shadow's relationship to objects: convexity, concavity, texture, etc. Arnheim (Arnheim, 1997) states that the gradients of brightness are the most efficient in creating the sense of depth, from landscapes to single objects.

Since the natural light (the Sun) comes from above, we tend to understand shadows under that condition. Studies in computational neuroscience suggest that the visual system is adapted to the statistical structure of its physical environment. That is why we understand the elements in Fig. 3 as convex and concave. If we rotate the Fig. 3 a) $180^{\circ}$, we obtain Fig. 3 b), in which we understand exactly the opposite!

Knowing that variation is fundamental to perception, we state the importance of the evolution of intrinsic shadows as an object is moved in relation to a light source: on the creation, destruction, merging, and splitting of shadows [Knill, et al., 
1997]. This variation could be important for better describing the volumetric characteristics of an object.

If we are interested in colour analysis we should be aware of the colour transformation due to the shadows, a feature very well known to the painters. In that case, we should take a picture without shadows, and with a standard colour separation card, to be able to reproduce it with rigor.
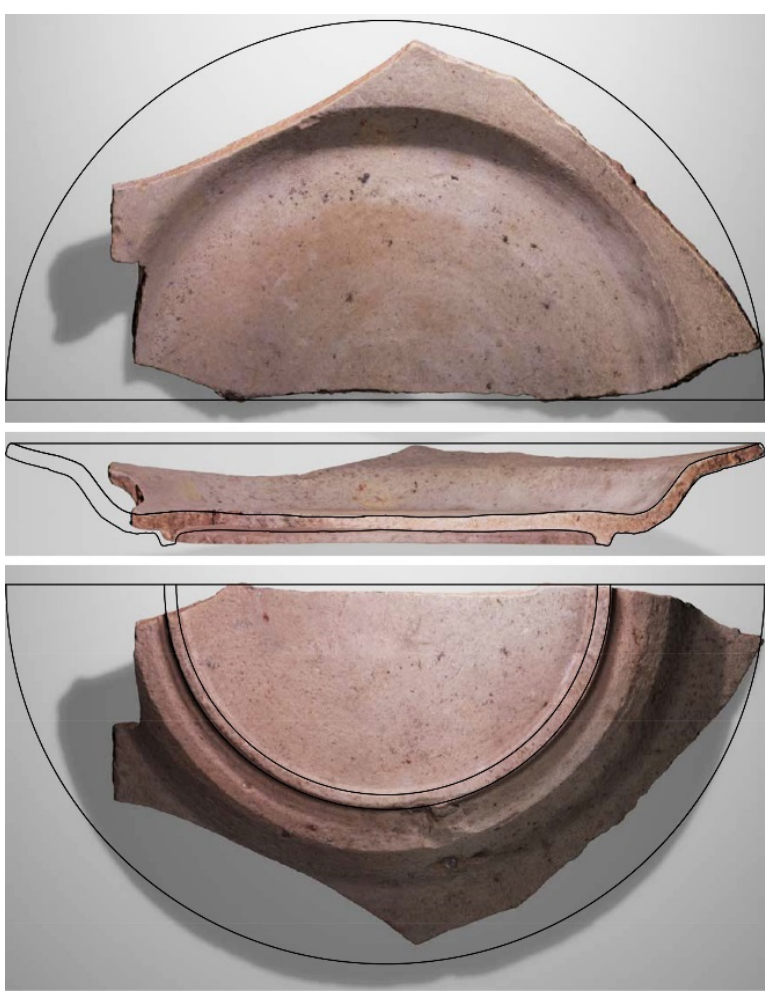

Figure 4: Orthometric representation of the plate, using cast and attached shadows.

Continuing our demonstration, we propose to apply both attached and cast shadow, using a light gray plane for representing the extrinsic shadow. As stated before, the better understanding of the object volumetric characteristics, is achieved placing the lighting source pointing downwards.

The renderization of shadows was conducted based on Reflectance transformation images built on Virtual Environment [García-Fernández, 2015]. Being RTIs a particularization of Shape-from-shading, in our specific case, the advantages of this approach are summarized in the capacities of real-time rendering and customization of the response to light stimuli. These allow us to test and compare different positions of the lighting source. The result is represented in Fig. 4.

\section{CONCLUSIONS}

The drawing of artefacts has always been a core activity in archaeology and finds in the digital technologies new opportunities to increase the clarity of the representation of the numerous artefacts retrieved from excavation. While shadows are usually eliminated from these representations, in favor of clarity of typologies and quickness and simplicity of the drawing, there is potential for the use of light and shade effects from photorealistic true-orthoimages of $3 \mathrm{D}$ models in the perception of concave/convex shapes. This is particularly pertinent for volumetric shapes such as plates, as was shown in this article, a quality it shares with other many other archaeological objects; it can also have further applications in the case of pieces with incised or applied decorations.

The considerations described in this paper demonstrate the feasibility to enhance the shape perception of artifacts with concave-convex predominance with the application of synthetic shadows pointing the light source downward and using the extrinsic cast shadows on the background, to be able to add information about the object's form. The representation here proposed, combining the aforementioned shadows with an orthometric representation, is intended to increase the understanding of the archeological artifacts, for both archaeologists and the non-expert public.

The partial results achieved on this study open new paths of study, especially in the representation of multiple light positions and its variation in multiple images, to enhance the geometrical understanding, and the analysis of the interference of the artifact's own colors and textures on the shadow's interpretation.

\section{ACKNOWLEDGEMENTS}

The research leading to these results has received funding from the Research and Innovation Framework Programme (Marie Curie Actions) of the European Union's Horizon 2020 Framework Programme H2020-MSCA-IF-2016, project 3DSMoHC no. 747046 .

\section{REFERENCES}

Adkins, L., Adkins, R., 1989. Archaeological Illustration. Cambridge: Cambridge University Press

Arnheim R., 1997, Visual Thinking, Berkeley, Los Angeles, London: University of California Press

Castro, A., Sebastian, L., 2003., A componente de desenho cerâmico na intervenção arqueológica no Mosteiro de S. João de Tarouca, In Revista Portuguesa de Arqueologia, Vol.6-2: 545-560.

Knill, D.C. et al., 1997, Geometry of Shadows, Jour-nal of the Optical Society of America, 14:12, pp 3216-3232, accessed 29 June 2018.

Lima, L., 2007. O Desenho como Substituto do Objecto: Descrição Científica nas Imagens do Dese-nho e Materiais Arqueológicos. Master Thesis,. Faculdade de Belas Artes da Universidade do Porto

Madeira, J. L., 2013, O desenho na Arqueologia, Coimbra: Instituto de Arqueologia da Faculdade de Letras, $2^{\text {a }}$ Edição

Manso, C., Medeiros, L., and Kruz, A., 2011. Relatório Final de Trabalhos Arqueológicos - Rua das Madres, $\mathrm{n}^{\circ} 59$, Lisboa: Atalaia Plural e DGPC

Martorelli, M., Pensa, C., Speranza, D., 2014, Digital photogrammetry for documentation of maritime heritage. Journal of Maritime Archaeology, 9(1), 81-93 
Niederoest, M., Niederoest, J., Scucka, J., 2004, Shape from Focus: Fully Automated 3D reconstruction and visualization of microscope Object, Institute of Geonics in Ostrava, pp. 45-76.

García-Fernández, J., 2014, Digital Culture for the enhacement of heritage Value, $\mathrm{PhD}$ Thesis, Ed. University of Valladolid, Spain

García-Fernández, J., 2015, 2.5D images based on reflectance information for the interpretation of architectural heritage, Revista De Teledeteccion, 2015(44)

Griffiths, N., Jenner, A., and Wilson, C., 1990. Drawing archaeological finds: a handbook, 1st ed. [S. 1.]: Archetype Publication

Stone et al., 2009, Where is the light? Bayesian perceptual priors for lighting direction. Proceedings of the Royal Society 276, pp. 1797-1804

Swirnoff, L., 1986, Dimensional Color. New York: Van Nostrand Reinhold.

Ullman, S., 1996, High-Level Vision: Object Recognition and Visual Cognition. London: MIT Press.

Castro, A., Sebastian, L., 2003., A componente de desenho cerâmico na intervenção arqueológica no Mosteiro de S. João de Tarouca, In Revista Portuguesa de Arqueologia, Vol.6-2: 545-560. 